\title{
Le juge doit-il appliquer une loi inique?
}

\section{Danièle Lochak}

\section{Le Genre Humain, $\mathrm{n}^{\circ} 28$, novembre 1994, « Juger sous Vichy »}

La question cruciale, la question fondamentale qui a servi de point de départ au présent volume, est celle-ci : que doit faire le juge par rapport à une loi inique ? Autrement dit : doit-il l'appliquer, doit-il ne pas l'appliquer, peut-il, au demeurant, ne pas l'appliquer ? Et ici, il s'agit moins de porter un jugement que de poser des questions. On a pu relever à cet égard la prudence, ou plutôt la modestie affichés par les futurs magistrats qui ont pris la parole : nous ne voulons pas nous ériger en juges de nos aînés, ont-il dit en substance, nous n'avons aucun titre pour les juger, nous voulons simplement comprendre et en tirer des leçons pour nous mêmes, pour l'avenir. Comme le rappelle Alfred Grosser*, il est toujours un peu facile de jouer les grandes âmes après coup, car on n'est jamais assuré de savoir la conduite qu'on aurait soi-même tenue, confronté au problème.

Il me semble, cependant, que ceux qui ont vécu et exercé des fonctions pendant cette période auraient pu, eux, réfléchir sérieusement et prendre plus clairement position, après la guerre, sur ce qui s'était passé, et ne pas hésiter, le cas échéant, à faire leur autocritique collective en tant que corps. L'attitude du Conseil d'Etat, à laquelle Jean Marcou fait allusion, et qui a été je pense celle de toutes les autres corporations (magistrature, facultés de droit, et sans doute bien d'autres encore que je connais moins bien), me paraît très contestable. Elles ont en général fait le silence sur ce qui s'était passé, voire, dans le cas du Conseil d'Etat, se sont reconstruit après coup une sorte de légitimité. Comme si leurs membres, même ceux qui n'avaient rien à se reprocher, même ceux qui avaient observé pendant la guerre et l'occupation une attitude honorable, même ceux qui avaient le cas échéant été d'authentiques résistants, hésitaient, par une sorte de réflexe de solidarité corporative, à remettre en cause l'institution à laquelle ils appartenaient, craignant que la critique de certains comportements passés ne rejaillisse sur l'ensemble du corps.

Si nous revenons à la question centrale, nous constatons que le juge se trouve placé devant une alternative en forme de dilemme : il doit normalement obéir aux lois de l'Etat et les appliquer, puisque telle est sa mission et qu'il ne doit pas se substituer aux autorités investies du pouvoir de faire la loi ; mais il y a un moment où le simple fait d'appliquer la loi, et donc de faire son métier, le rend complice d'actes moralement répréhensibles. Jusqu'à quand faut-il continuer à appliquer la loi, à quel moment faut-il cesser de l'appliquer ? On pourrait répondre que l'obligation d'appliquer la loi ne vaut qu'à l'intérieur d'un État de droit. Mais, comme l'a déjà montré Henry Rousso dans ses travaux, le problème qui s'est posé avec Vichy, c'est qu'il y a eu continuité apparente de l'Etat et du régime légal. Pétain ne s'est pas imposé par un coup d'Etat, il a été investi par un vote dont on a pu contester plus tard la légitimité, voire même la constitutionnalité, mais qui revêtait les formes extérieures de la légalité. La rupture entre la IIIe République et le régime de Vichy ne s'est fait pas opérée de façon brutale, mais subrepticement, à mesure qu'on été promulgués des textes qui prenaient le contrepied de la tradition républicaine, à commencer, puisque c'est évidemment le plus spectaculaire, par le statut des Juifs. Et si le Parlement a été dissous - ce qui, on peut l'admettre, n'est pas tout à fait un détail - toutes les institutions administratives et judiciaires sont restées en place, de même que sont pour l'essentiel restés en vigueur les lois et réglements antérieurs.

* Voir, dans ce même volume, p. 107

** Voir, dans ce même volume, p. 83 
À partir de quel moment, en faisant ou en croyant faire normalement son métier entre-t-on dans la voie de la compromission ? Un magistrat peut-il, au demeurant, faire normalement son métier dans un régime dont il réprouve les fondements politiques, idéologiques, moraux ? Peut-il résister en restant à son poste, ou doit-il à un moment donné faire le choix de démissionner ? À cet ensemble de questions cruciales je n'ai trouvé, à l'occasion de mes recherches, aucune réponse, ni même de tentative de réponse de la part de ceux qui, en tant que fonctionnaires, ont bien dû, bon gré mal gré, servir Vichy. Est-ce à dire qu'ils ne se la sont même pas posée ? En tout cas, s'ils l'ont fait, c'est dans leur for intérieur, puisqu'il n'en reste pas trace.

J'ai trouvé en revanche, chez des auteurs italiens qui ont vécu la période du fascisme, des réflexions sur cette question, en particulier dans les mémoires d'un magistrat, Alessandro Galante Garrone ${ }^{1}$. L'auteur explique comment en 1932 - bien après l'arrivée au pouvoir de Mussolini, par conséquent - il a choisi d'entrer dans la magistrature, pensant que c'était là qu'il serait le plus libre et le plus indépendant des pressions du régime, et qu'il pourrait le mieux défendre la légalité et les citoyens contre l'arbitraire, en appliquant des lois dont la plus grande partie datait d'avant l'installation du fascisme. L'exemple de quelques magistrats inflexibles montrait, dit-il, qu'il était possible de faire tranquillement son devoir sans se plier aux injonctions du pouvoir politique ; d'autant que le fascisme, après avoir fait taire brutalement les opposants et s'être imposé par la force, n'exigea pas, contrairement au régime nazi, une adhésion active et se contenta d'un consensus fictif. Progressivement, à partir de 1933, sous l'influence du nazisme, un discrédit croissant commença à entourer le droit, de sorte que la défense de la légalité se mua peu à peu, dit l'auteur, en une «affirmation polémique ». Mais le véritable tournant s'opéra en 1938, qui marque le «passage de la comédie à la tragédie », le début du « quinquennat apocalyptique », avec l'édiction des lois anti-juives.

Ces lois portèrent au degré suprême le dilemme devant lequel est placé le juge appelé à appliquer une loi qui moralement lui répugne. Jusqu'à quand doit-il continuer à défendre à outrance le principe de légalité, à appliquer imperturbablement la loi quel que soit le jugement personnel qu'il porte sur elle ? Si sa mission est d'appliquer toutes les lois, bonnes ou mauvaises, de façon impartiale, et en traitant identiquement les situations identiques, il arrive pourtant un moment où le juge, qui est aussi un citoyen, ne peut plus observer cette ligne de conduite sans rencontrer des problèmes de conscience : il tentera alors d'interpréter la loi de façon à en atténuer la portée, à la vider le plus possible de son contenu. Mais dans les hypothèses extrêmes - et tel était le cas de la législation raciale qui n'avait plus de la loi que l'habit hypocrite - cette solution est elle-même insuffisante. Pour remédier à l'injustice du législateur et corriger la monstruosité morale de la loi il n'y a plus d'autre alternative que d'opter pour l'illégalité... ce qui revient à se renier comme juriste et comme juge.

Mais ce choix lui-même, comment pouvait-il se concrétiser en pratique ? Garrone explique comment, à quelques-uns, autour de lui, les juges se concertèrent et arrivèrent à la conclusion qu'ils devaient d'une part faire prévaloir une interprétation stricte des textes de 1938 qui étaient en contradiction flagrante avec les principes fondamentaux de l'ordre juridique, d'autre part s'efforcer de saboter la législation antisémite en vue d'en restreindre au maximum les effets, par différents artifices. Mais, comme il le reconnaît, ces deux voies, pas toujours faciles à emprunter, étaient de surcroît d'une efficacité limitée pour paralyser l'application des lois raciales. Devant « la conscience de l'extrême modestie et de l'inanité de ces efforts », Garrone choisira finalement la voie de l'activité illégale et clandestine puis de la Résistance.

${ }^{1}$ Alessandro Galante Garrone, Amalek. Il dovere della memoria, Rizzoli, 1989. 
Si j'ai insisté longuement sur ce cas, ce n'est pas parce que son attitude est représentative de la magistrature italienne - elle ne l'est pas -, mais parce que Garonne analyse clairement les termes du dilemme. De l'expérience qu'il relate, on peut déduire que ce dilemme est sans issue, qu'il y a un moment où le juge n'a d'autre choix, s'il veut garder les mains absolument propres, que de démissionner. Je dis «absolument propres » pour pointer le fait que les magistrats qui sont restés à leur poste ne se sont pas ipso facto déshonorés, mais qu'ils ont pris le risque de se salir les mains à un moment ou à un autre, en ayant à appliquer - fût-ce en les interprétant de la façon la plus restrictive qui soit - les lois antisémites ou d'autres lois indignes.

\section{La responsabilité spécifique des juristes sous Vichy}

J'en viens maintenant à une autre question, qui concerne la part de responsabilité qui revient aux juges dans la mise en œuvre de la politique antisémite de Vichy. Je crois qu'il y a une responsabilité spécifique des magistrats ; je ne dis pas exclusive, ni même principale, mais spécifique. En effet, le régime de Vichy avait besoin de juristes en général et de juges en particulier. Il avait besoin de juges, en premier lieu, comme tous les régimes autoritaires qui tiennent à conserver les formes de l'État de droit : Mussolini lui-même a conservé le Conseil d'Etat sans lui ôter une seule de ses prérogatives en matière de contrôle de l'administration. Mais Vichy avait aussi besoin de juges pour faire appliquer la législation antisémite et lui donner une légitimité accrue. C'est sur ce point que porte la responsabilité spécifique des juristes et des juges.

Les juges n'ont pas pris de part directe dans la déportation et l'extermination des Juifs, ils n'ont en aucune façon collaboré à l'œuvre d'extermination, comme cela a été souligné à plusieurs reprises. Mais on ne peut pas pour autant dissocier entièrement les mesures d'exclusion qui ont frappé les Juifs à partir de 1940, lesquelles, en donnant lieu à du contentieux, ont impliqué les juges, de l'entreprise ultérieure d'extermination à laquelle ils n'ont pas pris part. S'il est vrai que la responsabilité principale de la déportation des Juifs n'incombe pas à Vichy, s'il est vrai également que l'anéantissement des Juifs n'était pas contenu en germe dans le statut des Juifs, il n'en demeure pas moins que, comme le montre Raul Hilberg dans La destruction des Juifs d'Europe, un enchaînement logique et inexorable relie entre elles les différentes étapes du processus de destruction : depuis la définition du Juif jusqu'à la «solution finale » en passant par les phases intermédiaires de l'expropriation et de la concentration dans des ghettos. C'est pourquoi la définition du Juif, qui fait figure de mesure relativement anodine, apparaît comme la condition préliminaire et indispensable à l'action ultérieure dans la mesure où elle permet de délimiter précisément la cible de cette action.

Or les magistrats ont apporté concrètement leur concours, comme nous le lisons dans les chapitres du présent volume, à la détermination de la qualité de «Juif ». Mais au-delà de ce concours concret, ils ont surtout contribué à asseoir la légitimité de la législation antisémite : en acceptant d'appliquer et d'interpréter les textes qui définissaient le «Juif », ils ont contribué à faire admettre comme évidente l'idée que les Juifs étaient d'une espèce - d'une « race »... différente, qu'ils nétaient pas des citoyens comme les autres, pas des sujets de droit comme les autres, et finalement pas des hommes comme les autres, qu'il était légitime de dépouiller de leurs droits et de leurs biens.

C'est là, à mon sens, que réside la responsabilité essentielle et spécifique des magistrats, même si, encore une fois, l'écrasante majorité des Juifs déportés n'ont jamais eu affaire à eux, et c'est donc sur ce point que je voudrais insister.

Peu importe à cet égard le sens des décisions rendues : loin d'être toujours défavorables aux Juifs, les juges n'hésitant pas le cas échéant à prendre le contrepied de la position du 
Commissariat général aux questions juives. Mais le simple fait de devoir appliquer les critères du législateur contraint à entrer dans la logique de l'antisémitisme «légal » et à se couler dans le système de pensée du législateur. Je cite un exemple qui me paraît particulièrement significatif des contorsions juridiques - si l'on peut les qualifier ainsi - auxquelles l'application de la législation antisémite conduit les juges. Dans une affaire jugée en 1941, dans laquelle le juge va finalement décider qu'un individu né de deux grands parents juifs, marié avec un non juif, mais qui ne s'est pas formellement converti à la religion catholique ou protestante, n'est pas juif, le rapporteur va se livrer à un long raisonnement dont j'extraie le passage suivant : " [Pour qu'il soit considéré comme juif], il faut que cet individu hybride prouve par son appartenance à la religion judaîque qu'il a conservé, malgré une origine mixte, un attachement tout spécial à la race juive. S'il n'est pas de confession juive, les quelques liens ancestraux qui le rattachent encore à la race juive s'évanouissent devant ceux qui l'attachent désormais à la race aryenne, liens ancestraux égaux et lien conjugal... Cet individu est dégagé de toute servitude raciale juive $»^{2}$. On ne peut s'empêcher de penser que cette décision favorable est payée au prix fort, et que le bénéfice individuel qu'en retire le requérant est largement contrebalancé au niveau collectif par la compromission avec l'idéologie antisémite des juges que reflète le passage du texte cité.

\section{Magistrat, juge et citoyen}

Une décision de justice ne vaut pas seulement pour l'individu à l'égard de qui elle est rendue. Je veux dire que, dans une décision de justice, il y a aussi une interprétation de la loi, et que cette interprétation, du moins lorsque l'État de droit fonctionne en respectant ses propres principes, s'impose à l'administration. Par conséquent, même si les décisions des magistrats n'ont concerné qu'une infime minorité de personnes, elles n'en ont pas moins fixé une interprétation, donné un mode d'emploi de la loi. Ainsi, lorsqu'une décision est favorable aux intéressés, elle limite d'autant pour l'avenir les pouvoirs de l'administration en lui imposant une interprétation restrictive de la loi ; inversement, une décision qui interprète largement les pouvoirs de l'administration lui délie les mains pour l'avenir. Voici donc une première raison pour laquelle le constat, évidemment imparable sur le plan des faits, selon lequel la quasi-totalité des Juifs qui ont été exterminés ne sont pas passés entre les mains de la justice, ne doit pas conduire à éluder la question de la responsabilité des magistrats.

La seconde raison renvoie à ce que l'effet nocif des décisions de justice, et de la pratique des juristes en général, résulte moins du contenu des décisions prises que de la façon de poser et de résoudre les questions, en se conformant à la problématique imposée par le législateur et en reprenant donc à son compte la problématique et les concepts de l'antisémitisme «légal ». Il me semble qu'il aurait mieux valu, finalement, y compris pour les Juifs eux-mêmes, que les juges refusent d'appliquer cette législation et que les commentateurs refusent de la commenter, fût-ce au prix d'une plus grande latitude d'action laissée au Commissariat général aux questions juives (CGQJ), plutôt que de consentir à l'appliquer, fût-ce en en proposant une interprétation restrictive susceptible de sauver quelques individus.

Au-delà des conséquences concrètes des décisions, il faut donc aussi, voire surtout, prendre en compte leurs effets symboliques et leur portée idéologique. Ce qui mène à s'interroger sur l'attitude à observer face à des lois qui, y compris dans un régime démocratique, portent des atteintes suffisamment graves aux libertés fondamentales pour altérer l'idée qu'on se fait d'un État de droit.

2Trib. civ. Rabat, 17 décembre 1941, JCP 1942.II.1800. 
L'idée, souvent avancée, selon laquelle le magistrat doit être fidèle à la loi, appliquer la loi avec loyauté, et non être fidèle au régime, il me semble que la distinction perd son sens, justement, dans le cas de Vichy. Quelle conduite suivre, dans ce cas ? C'est peut-être dans la distinction entre le rôle du magistrat comme juge et son rôle de citoyen que l'on peut trouver l'ébauche d'une réponse. A défaut de réagir individuellement, en «sabotant » l'application des lois, ce qui peut paraître un peu théorique lorsque l'on est pris dans un système, ils auraient pu au moins se manifester collectivement.

Dans cette optique, le reproche que l'on pourrait précisément leur faire, c'est de ne pas s'être manifestés collectivement alors qu'ils auraient pu, sans courir aucun risque, dire d'une seule voix : nous contestons ces lois. A vrai dire, je soupçonne qu'ils ne les contestaient pas vraiment et qu'à l'instar de la majorité des Français les lois anti-juives ne les ont pas choqués.

La grande majorité des juges n'étaient vraisemblablement pas hostiles aux mesures qui excluaient les Juifs de la fonction publique et de la magistrature. Si l'on admet cette vérité toute simple, la question de savoir pourquoi ils n'ont pas protesté devient sans objet. On sait qu'au Conseil d'État deux présidents de section ont été atteints par l'épuration antisémite : deux présidents de section sur cinq, cela fait $40 \%$, n'est-ce pas ? Combien étaient-ils pour penser qu'il y avait trop de Juifs au Conseil d'Etat et que l'épuration, après tout, n'était peut-être pas si injustifiée que cela?

Mais, indépendamment de cette dernière remarque, la réaction collective ne résoud pas la question fondamentale : qu'est-ce que je peux faire face à des lois inacceptables, moralement condamnables, en tant que magistrat qui, sauf à démissionner, dois juger, dois décider?

Seul face à la loi inique, que peut faire le citoyen en tant que magistrat ? A-t-il une autre alternative que démissionner ou appliquer la loi ? Démissionner est sans doute la solution la plus satisfaisante moralement, mais elle n'est pas généralisable : on ne peut ériger l'héroïsme en impératif catégorique. S'il ne démissionne pas, quelle marge de manœuvre lui-reste-t-il, puisque le juge ne peut pas refuser de juger ? C'est d'ailleurs ce qui fait que je serais moins sévère à leur encontre qu'à l'encontre des juristes - professeurs ou praticiens - qui ont commenté les lois antisémites, alors que, eux, n'y étaient absolument pas obligés. Ce magistrat, donc, qui n'a pas choisi de démissionner, qu'est-ce qu'il peut faire ? Seul, peu de choses, sauf intepréter la loi de la façon la plus restrictive possible, puisqu'il est enfermé dans un système qui lui enjoint de statuer. Mais en tant que citoyen, il retrouve la liberté, avec d'autres, de résister.

Cela dit, je pense qu'il serait utile de quitter à présent ces exemples extrêmes et de nous replacer dans le contexte de périodes moins exceptionnelles où les principes de l'Etat de droit sont grosso modo respectés.

\section{Et aujourd'hui?}

La question de savoir ce que doit faire un juge face à des lois qui lui semblent inéquitables ou qui vont à l'encontre de sa propre conception de l'équité n'est pas nouvelle. Je note que la discussion sur ce point a déjà été menée, notamment après 1968, au sein du Syndicat de la magistrature. La question n'est d'ailleurs pas simple, car le magistrat n'a pas de légitimité propre, et n'a donc pas de raison de faire prévaloir ses conceptions à lui sur celles du législateur qui, après tout, est censé exprimer la volonté générale. Il garde cependant la possibilité d'interpréter les lois dans le sens qui lui paraît le plus conforme à l'idée qu'il se fait de la justice : il prend simplement le risque d'être démenti en appel, et à terme d'être freiné dans son avancement. Car le poids de la hiérarchie est très fort dans l'institution judiciaire, beaucoup plus qu'au Conseil d'Etat 
où l'on avance à l'ancienneté. Mais s'il est prêt à prendre ce risque, il a sinon toute latitude pour statuer comme il l'entend, du moins une marge d'appréciation non négligeable.

Ouvrons une parenthèse sur la notion d'État de droit, qui doit être maniée avec précaution car elle peut être trompeuse. Après tout, Vichy était d'une certaine façon un Etat de droit si l'on se fonde sur des critères purement formels : il y avait des textes que l'on appliquait, il y avait même une hiérarchie des normes, puisque les lois, quoique adoptées par Pétain seul, avaient une valeur supérieure à celle des décrets, il y avait un juge pour censurer les actes administratifs illégaux. Aujourd'hui a fortiori, nous vivons dans un État de droit, avec de vraies lois votées par le Parlement, et même un Conseil constitutionnel qui peut les invalider lorsqu'elles ne sont pas conforme à la norme constitutionnelle suprême.

Mais cette conception purement formelle de l'Etat de droit rencontre assez vite des limites. Elle est commode, certes, car elle permet de se mettre d'accord assez facilement sur ce qu'implique l'État de droit : en gros, une hiérarchie des normes sanctionnées par un contrôle juridictionnel. Mais sur cette base minimale on peut fonder une législation répressive. On ne peut donc pas faire abstraction du contenu des lois pour apprécier l'existence d'un État de droit authentique.

Pour illustrer mon propos, je prendrai l'exemple des lois sur les étrangers, qui viennent, on le sait, d'être modifiées dans un sens particulièrement répressif. Ces lois, par rapport aux critères que j'ai énoncés, ne nous font pas sortir de l'État de droit : elles ont été votées par les représentants du peuple, elles ont été soumises au Conseil constitutionnel qui a validé l'essentiel de leurs dispositions. Quant à la disposition sur le droit d'asile qu'il a jugée non conforme à la Constitution, elle a pu être réintroduite dans la loi après qu'on ait convoqué les deux chambres du Parlement réunies en Congrès - et censées représenter le peuple souverain, seul détenteur du pouvoir constituant - afin qu'il révise la Constitution. Les formes de l'Etat de droit sont donc sauves.

Je ne prétends pas que l'existence de ces textes sur les étrangers nous a fait sortir du jour au lendemain de l'État de droit, encore moins qu'il y a une commune mesure entre ces textes et les lois de Vichy ; mais j'estime - je livre bien entendu ici un point de vue personnel - qu'il est permis d'en contester la légitimité au nom des valeurs - et non plus des formes - de l'Etat de droit et que la question peut par conséquent se poser de savoir quelle attitude les juges doivent adopter à leur égard.

La question est loin d'être théorique puisque la justice a été progressivement enrôlée dans la répression de l'immigration irrégulière. Le juge est donc de plus en plus souvent sommé d'intervenir, de façon d'ailleurs ambivalente, puisqu'il est à la fois un agent de la répression et théoriquement - le garant de la liberté individuelle, conformément à l'article 66 de la constitution. Or lorsqu'on considère les décisions rendues, force est de constater que les juges ont souvent tendance à oublier leur mission constitutionnelle pour faire prévaloir leur fonction répressive. Qu'il s'agisse des jugements rendus en matière de séjour irrégulier ou d'infractions à la législation sur les stupéfiants, ce qui frappe, c'est la sévérité des tribunaux correctionnels, qui prononcent avec une facilité déconcertante des peines d'interdiction du territoire dont l'effet, lorsqu'elles visent des étrangers ayant leurs attaches familiales en France, équivaut à un véritable bannissement. De même, lorsqu'un étranger est placé en rétention dans l'attente d'être reconduit à la frontière, il est prévu qu'un magistrat délégué par le président du tribunal de grande instance doit intervenir pour autoriser la prolongation de la privation de liberté au-delà de 24 heures. Jusqu'à la réforme récente, ils avaient le choix entre autoriser cette prolongation, assigné l'intéressé à résidence, ou le remettre en liberté. Or dans la quasi-totalité des cas, alors même que 
la loi prévoyait que la prolongation de la rétention devait être exceptionnelle, le juge optait malgré tout pour cette solution. Ne s'agit-il pas d'une démission pure et simple, d'un refus d'exercer la mission (que la Constitution lui assigne) de garantir la liberté individuelle ? Désormais la loi ne prévoit plus la possibilité de remettre l'intéressé en liberté : elle semble ne laisser d'autre choix au juge que la prolongation de la rétention, ou, à titre exceptionnel et s'il a des documents d'identité, l'assignation à résidence. Or certains juges ont estimé qu'à partir du moment où la Constitution érige le juge judiciaire en gardien de la liberté individuelle, la loi ne peut, si les mots ont un sens, lui interdire de remettre en liberté la personne qui lui est présentée. Ils ont donc fait prévaloir leur mission constitutionnelle sur la lettre de la loi, prononcé des mises en liberté, au prix d'un conflit ouvert avec l'administration préfectorale.

Il me semble qu'il y a là, dans un contexte beaucoup moins tragique que la période de Vichy, un bon exemple des problèmes auxquels ils vont être concrètement confrontés et qui requiert donc autre chose qu'une réponse abstraite.

Je crois que les réactions collectives, fondées sur une réflexion en commun, sont à cet égard importantes. Le Syndicat de la magistrature a constitué à une certaine époque un lieu de réflexion; cette époque est sans doute révolue, mais cela n'empêche pas de créer d'autres instances où l'on peut réfléchir sur un certain nombre de problèmes du type de ceux que nous posons aujourd'hui et définir ensemble une ligne de conduite.

Par ailleurs, il faut aussi ne pas se laisser enfermer dans le carcan de l'interprétation donnée par les juridictions suprêmes, ne pas hésiter à contredire les interprétations acquises. Après tout, la jurisprudence s'élabore à partir des décisions des juridictions de première instance, et face à une pratique judiciaire constante des juges de base il n'est pas exclu que les cours d'appel finissent par suivre à leur tour. Si les juges de première instance n'avaient pas peur de voir leurs décisions annulées, ils prendraient peut-être des décisions plus courageuses, plus inventives surtout.

Pour prendre ces décisions innovatrices, ils peuvent d'ailleurs aujourd'hui se fonder sur la Constitution elle-même (c'est l'exemple que j'ai donné tout à l'heure à propos du contrôle de la rétention adminsitrative), et plus souvent encore sur les conventions internationales.

Dès lors peut se poser la question suivante : les magistrats doivent-ils être porteurs de valeurs ? Là encore, nous nous trouvons face à un dilemme. Si l'on adopte une attitude positiviste, qui est spontanément celle du juriste pour qui la loi positive est la seule valide, on est conduit à appliquer cette loi et elle seule. Sinon, vous tombez vite dans les errements du jusnaturalisme. Pourquoi dis-je «errements » ? Parce que la référence au droit naturel n'est pas sans danger : si le droit naturel était porteur de potentialités progressistes à la Révolution française, il peut aussi servir à la promotion des valeurs les plus conservatrices : n'est-ce pas au nom du droit naturel - du droit naturel de l'embryon à la vie - que l'on dénie aux femmes le droit d'avorter ? Chacun a tendance à faire passer ses propres valeurs pour des principes immuables du droit naturel. Il faut donc manier prudemment l'idée que le droit est porteur de valeurs : le droit et le juste ne sont pas réductibles l'un à l'autre, ils ne peuvent pas et ne doivent pas être confondus parce que nous n'avons pas tous la même conception du juste.

Et les juges n'ont aucune légitimité pour faire prévaloir vos valeurs sur la loi écrite, qui, dans une démocratie, est réputée exprimer les valeurs auxquelles adhère le peuple.

Nous sommes tous, nous juristes, pris à l'intérieur de ce dilemme. Mais aujourd'hui nous avons peut-être une façon d'en sortir : grâce au développement de la protection internationale des droits de l'homme, nous avons de plus en plus souvent la possibilté d'écarter l'application de la 
loi interne non pas au nom de nos valeurs subjectives mais en faisant tout simplement prévaloir les normes internationales sur les normes internes. 